\title{
Planning of Maintenance Operations for a Motorway Operator based upon Multicriteria Evaluations over a Finite Scale and Sensitivity Analyses
}

\author{
Céline Sanchez ${ }^{1}$, Jacky Montmain ${ }^{2}$, Marc Vinches ${ }^{2}$ and Brigitte Mahieu ${ }^{1}$ \\ ${ }^{1}$ Service Structure Viabilité Sécurité, Société des Autoroutes Estérel Côtes d'Azur Provence \\ Alpes, avenue de cannes, 06211 Mandelieu Cedex, France \\ \{cesanchez, bmahieu\} @escota.net \\ ${ }^{2}$ Ecole des Mines d'Alès, 6 avenue de Clavières, 30319 Alès Cedex, France \\ \{jacky.montmain, marc.vinches\}@ema.fr
}

\begin{abstract}
The Escota Company aims at the formalization and improvement of the decisional process for preventive maintenance in a multi criteria (MC) environment. According to available pieces of knowledge on the infrastructure condition, operations are to be evaluated with regards to (w.r.t.) technical but also to conformity, security and financial criteria. This MC evaluation is modelled as the aggregation of partial scores attributed to an operation w.r.t. a given set of $n$ criteria. The scores are expressed over a finite scale which can cause some troubles when no attention is paid to the aggregation procedure. This paper deals with the consistency of the evaluation process, where scores are expressed as labels by Escota's experts, whereas the aggregation model is supposed to deal with numerical values and cardinal scales. We try to analyse this curious but common apparent paradox in $\mathrm{MC}$ evaluation when engineering contexts are concerned. A robustness study of the evaluation process concludes this paper.
\end{abstract}

Keywords. Multi-criteria decision-making, Multi-criteria aggregation, Finite scale, Decision support system, Motorway infrastructure.

\section{Escota Decision Process}

\subsection{Context}

The Escota Company, founded in 1956, is the leading operator of toll motorways in France. Due to its integration into the Provence-Alpes-Côte d'Azur region, Escota is committed, as every motorway operator, to a sustainable development approach, including the social, economic and environmental aspects of its activities. Every year, specific initiatives are undertaken, or repeated, to include the motorway network in a sustainable development approach. Within this scope, the Escota Company aims at the formalization and improvement of the decisional process for preventive maintenance and property management with the desire to show transparency on decisions relative to property management, personal accountability and justification of decision-making logic in a multi actors and multi criteria (MC) environment [6], [7]. These decisions concern upkeep, improvement and upgrading operations, 
involving technical, conformity, security or financial criteria. The operations are related to operating domains such as constructive works, carriageways, vertical roadsigns and carriageway markings, buildings, prevention of fire risks, open spaces... Managing such a complex infrastructure necessitates a dynamic Information Processing System (IPS) to facilitate the way decision-makers use their reasoning capabilities through adequate information processing procedure.

\subsection{Valuation of the Infrastructure Condition}

Periodic inspections are performed to detect and measure, as early as possible, any malfunction symptoms affecting an element of the infrastructure (EI). The expert in charge of an operating domain then analyses the technical diagnosis relative to the EI. He evaluates the situation seriousness in terms of technical risk analyses. This evaluation relies on a specific set of $n$ criteria relative to his domain. An aggregation with a weighted arithmetic mean (WAM) is then performed to assess a global degree of emergency to the corresponding maintenance operation. This evaluation is then submitted to the official in charge of the operating network. This latter coordinates the experts' needs and demands for operation planning purposes.

This paper deals more particularly with the MC evaluation process by the expert of an operating domain, i.e. the affectation of an emergency degree to an operation. There exist several methods to identify and perform aggregation process with a WAM. The Analytic Hierarchical Process, AHP, is probably the most famous one in industry [1]. However, because it explicitly guarantees the consistency between the commensurable scales it aggregates and the WAM operator it identifies, the Measuring Attractiveness by a Categorical Based Evaluation TecHnique method, MACBETH, has got recent successes [2], [3]. In our application, MACBETH is first used to build the valuation scale associated to each emergency criterion of a domain. It is then applied to determine the WAM parameters.

Furthermore, the way experts give their assessment in natural language raises another problem [4]. These labels are commonly converted into numerical values to perform the aggregation process. No particular attention is generally paid to this "translation". However the consequences over the aggregation results are damageable. In civil engineering, the culture of numbers is strongly developed. People commonly manipulate symbolic labels but may convert them into more or less arbitrary numerical values when necessary without further care. This cultural viewpoint explains why an aggregation operator is generally preferred to a rule base whereas appraisals are expressed in terms of symbolic labels [4]. A completely symbolic evaluation over finite scales could be envisaged [5].

Let us illustrate the scales problem with the following example. Let us suppose that the semantic universe of an expert w.r.t. the seriousness of a symptom is: \{insignificant, serious, alarming $\}$. We can imagine that a corresponding possible set of discrete numerical values (in $[0 ; 1]$ ) could be: $\{0 ; 0.5 ; 1\}$. There are several assumptions behind this translation concerning the nature of the scale. This point will be discussed later. Let us just note here that the numerical values are commonly chosen equidistant. Now let us consider another semantic universe: \{insignificant, minor, alarming $\}$. This time, the associated set of numerical values $\{0 ; 0.5 ; 1\}$ intuitively appears more questionable. The expert should prefer $\{0 ; 0.25 ; 1\}$. When seriousness degrees of several symptoms are to be aggregated, the result of the WAM 
aggregation strongly depends on the choice of the set of numerical values. Furthermore, in any case, the numerical WAM value does not necessary belong to $\{0$; $0.5 ; 1\}$ or $\{0 ; 0.25 ; 1\}$. It must then be converted into the convenient label in return.

The way labels are converted into numerical values (and back) coupled to the commensurability of the scales of the dimensions to be aggregated can entail serious problems when aggregating without any care. In this paper, we propose a methodology to build finite partial valuation scales consistently with WAM aggregation.

The paper is organized as follows. Some considerations are given about the way continuous cardinal scales are constructed with the Escota operating domain experts. Then, it is explained how to build a WAM aggregation operator w.r.t. each operating domain, in order to be consistent with the identified scales. The MACBETH method is the support of these first two steps. The problem related to the finite scales, that the experts use when assigning partial scores to an operation, is then considered. A method is proposed to ensure a logically sound interface between symbolic assessments and numerical computations in the framework of WAM aggregation. Then, a robustness analysis is proposed to determine the potential causes of overestimation or underestimation in the evaluation process of an operation.

\section{Cardinal Scales of Emergency Degress}

\subsection{Nature of Scales}

The purpose of this section is to explain how we have worked with Escota experts of the different operating domains in order to properly identify their emergency scales. There are one emergency scale for each criterion of the domain and one scale for the aggregated emergency value. In the following we will consider the case of the operating domain "carriageway". Eight criteria $(n=8)$ are related to it: security, durability, regulation, comfort, public image, environment protection, sanitary and social aspects.

It has been checked a priori that Escota emergency scales are of cardinal nature: the emergency scale relative to any of the criteria is an interval scale.

Let us consider a finite set $X$. When the elements of $X$ can be ranked w.r.t. to their attractiveness, this is ordinal information. It means that a number $n(x)$ can be associated to any element $x$ of $X$ such that:

$$
\begin{aligned}
& \forall x, y \in X:[x \mathrm{P} y \Leftrightarrow n(x) \succ n(y)] \\
& \forall x, y \in X:[x \mathrm{I} y \Leftrightarrow n(x)=n(y)]
\end{aligned}
$$

where relation $P$ «is more attractive than $»$ is asymmetric and non transitive and relation $I$ «is as attractive as» is an equivalence relation. $n(x)$ defines an ordinal scale.

Based upon this first level of information, an interval scale can then be built. The next step consists in evaluating the difference of intensity of preference between elements of $X$. It implies the following constraints: 


$$
n(x)-n(y)=k \alpha, k \in \mathrm{N}
$$

where $k$ characterizes the intensity of preference and $\alpha$ enables to respect the limits of the domain (for example [0,1]). The resolution of a system of equations of type (1), (2) and (3) provides an interval scale. That's the principle used in the MACBETH method [2].

\subsection{Emergency Scales and MACBETH Method}

The problem of commensurability of the dimensions to be aggregated is at the heart of the MACBETH method. Aggregation can be envisaged only if the scales relative to the emergency criteria are commensurable [3]. Then, MACBETH guarantees the consistency between the resulting partial scales and the WAM aggregation [2].

First, a training set of operations is constituted. A ranking of the operations in terms of emergency is established w.r.t. each criterion. At this stage, information is purely ordinal. Then, for each criterion, the solutions are compared pair to pair. Two fictive alternatives are introduced in the comparison process; they provide the reference values corresponding to the two emergency degrees: zero and one. The zero (resp. one) emergency degree corresponds to the threshold value under which operations are considered as not urgent at all (resp. highly urgent). The comparison then consists in quantifying the difference of emergency degree for each criterion. This difference is expressed in a finite set of labels: for example, "equivalent", "weak", "strong" and "extreme". The resulting set of constraints defines a linear programming problem. The solution of this problem provides the cardinal scale of emergency associated to one criterion. This step is repeated for each criterion.

Fig. 1 illustrates this process for criterion security. The carriageway expert compares 10 operations $\{\mathrm{A} \ldots \mathrm{J}\}$ pair to pair. The real names of operations are not given for confidentiality reasons. Two fictive operations urgent (highly urgent) and peu_urgent (not urgent at all) complete the training base. The "positive" label in Fig. 1 introduces a more flexible constraint because it simply replaces any label with a higher degree than weak. The resulting cardinal scale is given at the right side of Fig. 1.

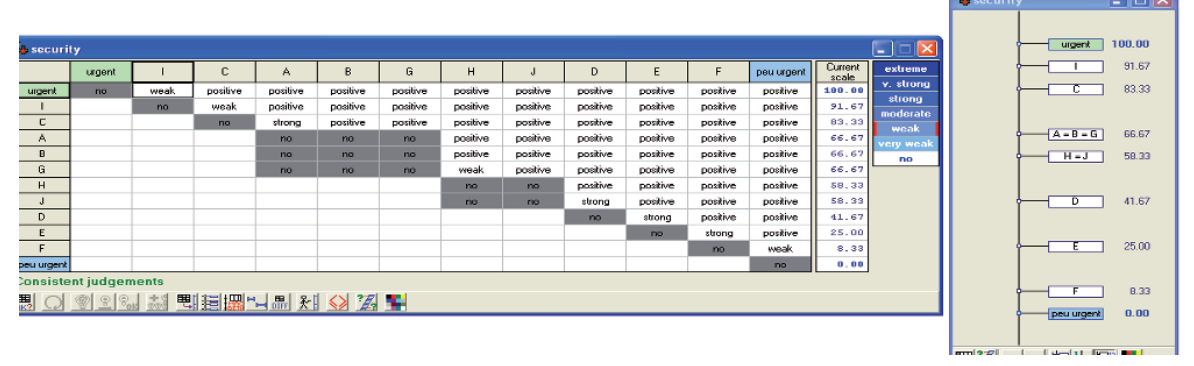

Fig. 1. MACBETH - Pair to pair comparison of operations and cardinal scale for security criterion.

Finally, this procedure is then applied to identify the weights of the WAM operator. The pair to pair comparison is carried out over the eight criteria of the carriageway domain (Fig. 2). The resulting interval scale of weights is given in Fig. 2. Let us note the 
weights $p_{i}, i=1 . . n$ ( $n=8$ for the carriageway domain). At this stage of the modelling, the carriageway expert has identified his 8 emergency scales and his WAM parameters. $\mathrm{He}$ is supposed to be able to compute the global degree of emergency of any operation when partial quotations $u_{i}$ are available, w.r.t. each criterion:

$$
\operatorname{WAM}(O P)=\sum_{i=1}^{n} p_{i} u_{i}
$$

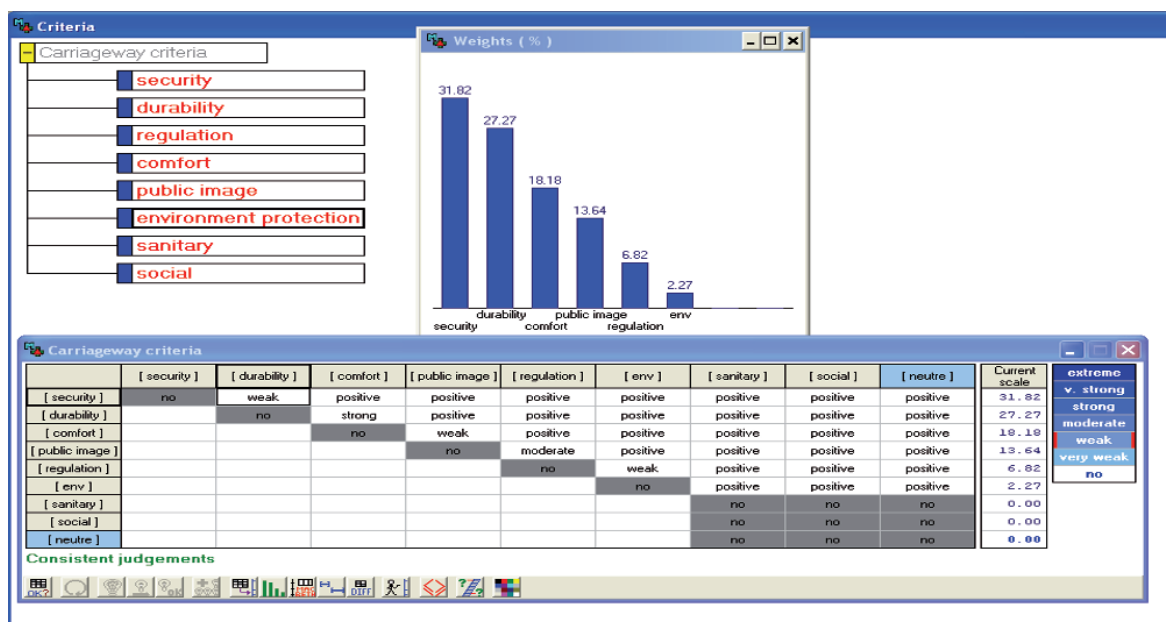

Fig. 2. MACBETH - Pair to pair comparison of carriageway criteria and weights identification.

\section{Discrete Cardinal Scales of Emergency}

Partial scores aggregation does not cause any problem when quotations referred to continuous cardinal scales. As explained in section 1, it is more questionable when partial scores are expressed on a discrete or finite scale. Indeed, Escota experts express their assessment w.r.t. each criterion on a finite set of 3 labels $\left\{U_{1}, U_{2}, U_{3}\right\}$. The different $\mathrm{U}_{\mathrm{i}}$ define a discrete cardinal scale. However, computing the WAM value necessitates assigning numerical values to each $U_{i}$. In the following, we describe the way this assignment can be achieved in a consistent manner with previous MACBETH identification phases.

A continuous cardinal scale has been identified with MACBETH method for the emergency scale of each criterion. The problem is now to assign a set of numerical values $\left\{u_{1}^{i}, u_{2}^{i}, u_{3}^{i}\right\}$ to $\left\{U_{1}, U_{2}, U_{3}\right\}$ for criterion $i$. Let us suppose the continuous cardinal scale for criterion $i$ has been identified with a training set of $q$ operations. These operations are grouped into 3 clusters corresponding to $U_{1}, U_{2}, U_{3}$. The computation of the clusters and their associated centres is achieved by minimizing the quadratic difference $\sum_{k=1}^{3} \sum_{j=1}^{q_{k}}\left(u_{k}^{i}-u^{i}\left(O P_{j}\right)\right)^{2}$ where $q_{k}$ is the number of operations in 
class $U_{k}\left(\sum_{k=1}^{3} q_{k}=q\right)$ and $u^{i}\left(O P_{j}\right), j=1 . . q$, the emergency degree of an operation $O P_{j}$ computed with MACBETH (Fig. 1).

In the example of Fig. 1, the computation of clusters gives: $u_{1}^{\text {security }}=0.91$, $u_{2}^{\text {sec urity }}=0.52$ and $u_{3}^{\text {sec urity }}=0.11$.

This assignment is repeated for each criterion relative to the carriageway domain. Then, the WAM can be numerically computed:

- For each criterion $i, i=1 . . n(n=8)$, a value $U_{k}$ is affected to an operation $O P$. Let us note this emergency degree $U_{k(i)}$;

- $O P$ is thus described by its vector of emergency degrees $\left[U_{k(1)}, . ., U_{k(n)}\right]$;

- The corresponding vector of numerical values is: $\left\{u_{k(1)}^{1}, u_{k(2)}^{2}, . ., u_{k(n)}^{n}\right\}$;

$$
W A M(O P)=\sum_{i=1}^{n} p_{i} \cdot u_{k(i)}^{i}
$$

The last constraint to be satisfied is that the WAM values must be converted in return into the semantic universe $\left\{U_{1}, U_{2}, U_{3}\right\}$. The output of the WAM operator must be discretized in $\left\{U_{1}, U_{2}, U_{3}\right\}$. The problem is thus to determine the centres of the $U_{k}$ clusters of the aggregated emergency scale (WAM values).

Let us note that the WAM operator is idempotent. Therefore, we must have:

$$
\forall U_{k}, k \in\{1,2,3\}, \operatorname{WAM}\left(U_{k}, \ldots, U_{k}\right)=U_{k}
$$

A sufficient condition for (5) is that the centres of the $U_{k}$ clusters of the aggregated emergency scale are the images of the corresponding $U_{k}$ centres of the partial emergency scales by the WAM function, i.e.:

$$
W A M\left(u_{k}^{1}, . ., u_{k}^{n}\right)=\sum_{i=1}^{n} p_{i} \cdot u_{k}^{i}=u_{k}^{A g}
$$

where $u_{k}^{A g}$ is the centre of class $U_{k}$ in the aggregated emergency scale.

Consequently, when an operation is defined by its partial emergency vector $\left[U_{k(1)}, . ., U_{k(n)}\right]$, equation (4) provides the numerical value

$$
W A M(O P)=\sum_{i=1}^{n} p_{i} u_{i}
$$

Then, the attribution of a class $U_{k}$ in the aggregated emergency scale is obtained through the following calculation:

$$
\min _{k}\left|u_{k}^{A g}-\sum_{i=1}^{n} p_{i} \cdot u_{k(i)}^{i}\right|
$$


The value of $k$ in $\{1,2,3\}$ that minimizes the expression in (8) provides the class $U_{k}$ of operation $O P$.

Fig. 3 summarizes the whole evaluation process of an operation $O P$. The validation of this process has been carried out with a test base of 23 operations in the carriageway domain. The carriageway expert has analysed each of these operations. For each of them, he has attributed emergency degrees in the Escota normalized semantic universe $\left\{U_{1}, U_{2}, U_{3}\right\}$ w.r.t. every of his 8 criteria.

Then, the aggregated emergency degree in this semantic universe can be computed using the 3-step process described in this paper (white arrows in Fig. 3). Besides these computations, the expert has been asked to directly attribute an overall emergency degree to each of the 23 operations (grey arrow in Fig. 3).

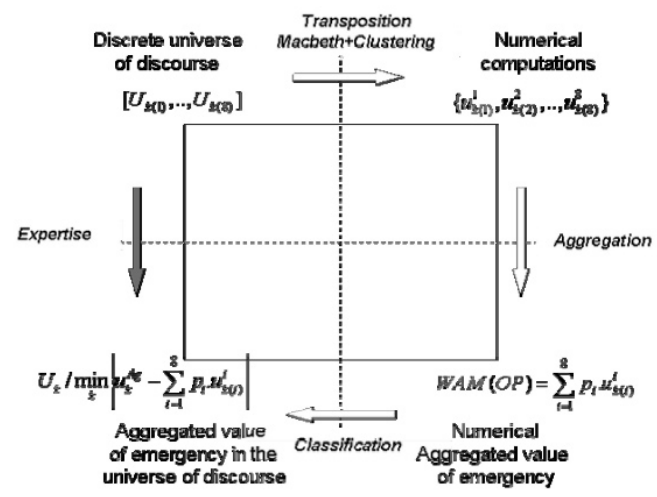

Fig. 3. Evaluation process of an operation.

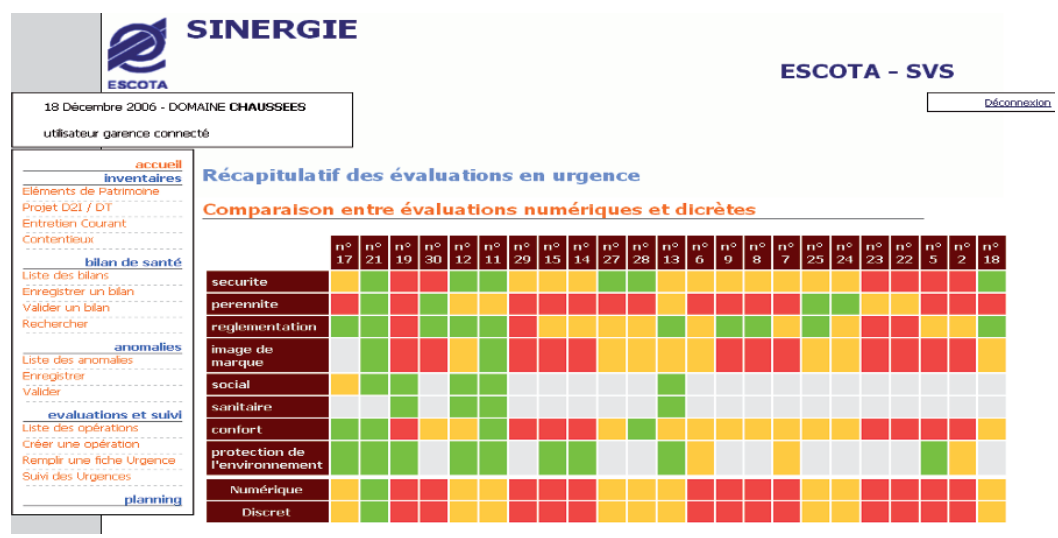

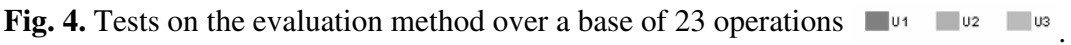

Fig. 4 reports these data. The last line corresponds to the direct expert evaluation (grey arrow). The last but one line provides the corresponding computed values with the 3-step method (white arrows). No error has been observed. However, the poor 
semantic universe - only 3 labels_-implied in our application can also partly explain such a perfect matching.

\section{The MC Hierarchical Evaluation by Escota}

In this paper, the study was focused on the MC evaluation by the expert of an operating domain. However, as evocated in section 1, planning of operations, by Escota, is more complex. The emergency assessment by operating domain experts described here is only part of a hierarchical MC evaluation process. From symptoms detection on elements of infrastructure to operation planning, a similar MC evaluation is carried out at different functional levels in the Escota organization.

The complete information processing used for Escota preventive maintenance can be formalized as the following sequence of risk analysis. Periodic inspections are performed to detect and measure any malfunction symptoms as early as possible. The expert in charge of a domain then analyses these technical diagnoses and evaluates the situation seriousness. The official in charge of the operating network coordinates and ponders the experts' needs and demands. Each actor of this information processing system participates to a tripartite MC decision-making logic: measurement, evaluation and decision. To each step of this process corresponds a specific set of criteria and an aggregation operator: seriousness of a malfunction results from a prescribed aggregation of the symptoms quotation; the expert's interpretation of the diagnosis associates an emergency degree to the corresponding maintenance operation w.r.t. the criteria relating to his operating domain (technical risks assessment); finally, the manager attributes a priority degree to the operation on the basis of a set of more strategic criteria (strategic risks analysis).

This hierarchical MC evaluation process enables to breakdown the decision-making into elementary steps. Each step collaborates to the enrichment of information from measures to priority degrees and thus contributes to the final step, i.e. operation planning.

We have developed a dynamic Information Processing System (IPS) to support this hierarchical MC evaluation of the infrastructure condition and facilitate the way decision-makers use their reasoning capabilities through adequate information processing procedure. Fig. 5 illustrates the man machine-interface the expert has at his disposal to fulfil an emergency form relative to an operation.

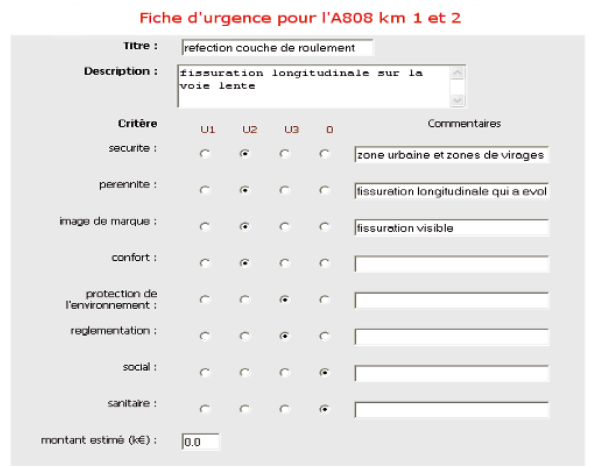

Fig. 5. Keyboarding of an emergency form. 
Finally, the emergency evaluation synthesis (Fig. 6) can be consulted by the official in charge of the operation network before he proceeds to his own MC evaluation.

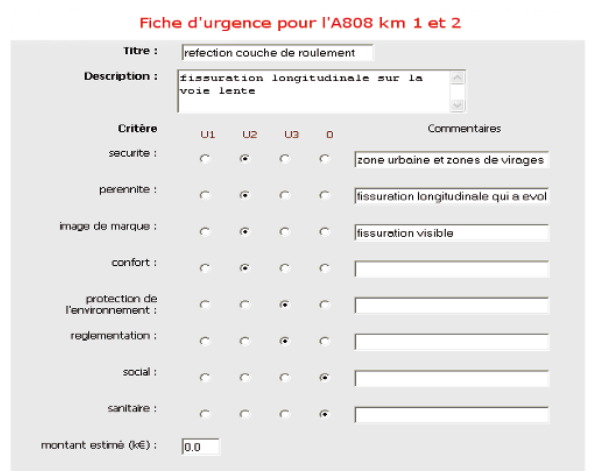

Fig. 6. Emergency evaluation synthesis.

\section{The Robustness Analysis of the Evaluation Process}

Let us now consider a last step in the evaluation process: assessment of the risk of erroneous estimation w.r.t. the emergency of an operation, i.e., the risk of underestimation or overestimation of the aggregated emergency score of an operation. It relies on a robustness analysis of the evaluation procedure based upon the WAM. Two aims are assigned to this step, it must answer the following questions: 1) when an erroneous partial estimation is done w.r.t. criterion $i$, what is the risk the aggregated emergency degree to be affected? 2) when an operation appears to be underestimated (resp. overestimated), which criteria could most likely explain this faulty result? The first question corresponds to an a priori risk estimation of erroneous evaluation; the second question is related to a diagnosis analysis.

Let us first define the notion of neighbourhood of a vector of emergency degrees $\left[U_{k(1)}, . ., U_{k(n)}\right]$ associated to an operation $O P$. The vectors of the neighbourhood of $\left[U_{k(1)}, . ., U_{k(n)}\right]$ are all the vectors $\left[U_{k(1)}^{\prime}, . ., U_{k(n)}^{\prime}\right]$ such that: $\forall i \in\{1 . . n\}, U_{k(i)}^{\prime}=U_{k(i)}$ or $U_{k(i)}^{\prime}$ is the value just above (resp. below) $U_{k(i)}$ (when defined; indeed, there is no value below zero and no value above $U_{1}$ ). The neighbourhood is a set of vectors denoted $\mathrm{N}\left(\left[U_{k(1)}, . ., U_{k(n)}\right]\right)$. In the example in dimension 2 in Fig. 7, $U_{k(1)}=U_{2}$ and $U_{k(2)}=U_{2}$. The values of component $i(i=1$ or 2$)$ of a neighbour vector may be $U_{2}$, $U_{1}$ or $U_{3}$. There are 8 neighbours. In the general case, the maximal number of neighbours is $3^{n}-1$. 


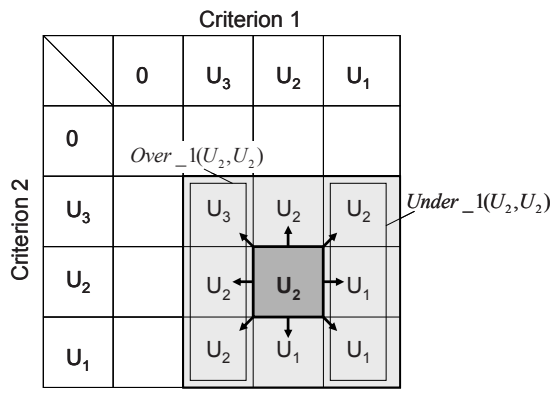

Fig. 7. Neighbourhood of the vector of emergency degrees $\left(U_{2}, U_{2}\right)$ in dimension 2 .

\subsection{Risk of Erroneous Estimation}

The risk of misclassification of an operation due to an overestimation (resp. underestimation) w.r.t. a criterion $i$ enables the expert in charge of a domain to assess the impact of an evaluation error w.r.t. criterion $i$ on the overall emergency degree of the operation. The higher, the more carefully the partial appraisal w.r.t. criterion $i$ must be carried out. The lower, the weaker the impact of the criterion to the global emergency degree. The risk analysis is based upon the following algorithm. We'll first consider the risk of underestimation for sake of simplicity. We consider that a value $U_{k(i)}$ is underestimated (resp. overestimated) when it should take the value just above $U_{k(i)}$ (resp. just below $U_{k(i)}$ ). This assumption means that the worst appraisal error w.r.t. one criterion can only correspond to the value just below or just above for this criterion.

Let's consider a vector $U=\left[U_{k(1)}, . ., U_{k(n)}\right]$

Compute $W A M(U)$

For each criterion $i$ :

- Find all the vectors $U^{\prime}=\left[U_{k(1)}^{\prime}, \ldots, U_{k(n)}^{\prime}\right]$ in $\mathrm{N}(U)$ such that $U_{k(i)}^{\prime}$ takes the value just above $U_{k(i)}$ (when defined, else $U_{k(i)}=U_{1}$ and there is no risk of underestimation w.r.t. criterion $i$ in this case). Note this set: $U_{n d e r} i(U)$

- Count the numbers of vectors $U^{\prime}$ in Under_i(U) such that $W A M\left(U^{\prime}\right)$ is higher than $W A M(U)$. Note this number $n_{\text {under }} i$

- The risk of underestimation induced by criterion $\mathrm{i}$ for an operation characterized

by $U$ is then:

$$
\text { risk_under }(i)=\frac{n_{\text {under_i }}}{\mid \text { Under_i(U)| }}
$$

In the example in Fig. 7, let us consider an assumption of underestimation w.r.t. criterion 1. The set Under $1\left(U_{2}, U_{2}\right)$ is represented in the figure. $\mid$ Under_ $1\left(U_{2}, U_{2}\right) \mid=3$; only $\left(U_{1}, U_{2}\right)$ and $\left(U_{1}, U_{1}\right)$ lead to an overall underestimation 
(the operation is evaluated $U_{2}$ whereas it should be $U_{1}$ ). Then, $n_{\text {under }_{-}}=2$ and risk_under $(1)=2 / 3$. It means that an underestimation w.r.t. criterion 1 for an operation characterized by $\left(U_{2}, U_{2}\right)$ leads to an underestimation of the overall degree of emergency of the operation in $66 \%$ of the cases.

The algorithm is the same for the risk of overestimation. Nevertheless, in this case, when $U_{k(i)}=0$, the risk of overestimation w.r.t. criterion $i$ is null. Fig. 8 and Figure 9 provide the results for the risk analysis when underestimation (Fig. 8) and when overestimation (Fig. 9) for all the vectors in Fig. 4.

\subsection{Diagnosis Analyses}

When the degree of emergency of an operation is suspected to be overestimated (resp. underestimated), the diagnosis analysis consists in determining the most likely causes, i.e., the criteria that the most frequently entail an overestimation (resp. underestimation) of the operation when they're overestimated (resp. underestimated) themselves. The possibility that criterion $i$ is a cause of overestimation (resp. underestimation) assuming an overestimation (resp. underestimation) of the overall emergency degree of the operation-is computed in the diagnosis step.

Let us consider the algorithm in case of underestimation (resp. overestimation).

Let's consider a vector $U=\left[U_{k(1)}, . ., U_{k(n)}\right]$

Compute $W A M(U)$

Compute $\mathrm{N}(U)$ and its cardinal $|\mathrm{N}(U)|$

- Compute $W A M\left(U^{\prime}\right)$ for each $U^{\prime}=\left[U_{k(1)}^{\prime}, . ., U_{k(n)}^{\prime}\right]$ in $\mathrm{N}(U)$

- Let us note Higher_ $\mathrm{N}(U)$ (resp. Lower_ $\mathrm{N}(U)$ ), the set of vectors $U^{\prime}$ in $\mathrm{N}(U)$ such that $W A M\left(U^{\prime}\right)>W A M(U)\left(\right.$ resp. WAM $\left.\left(U^{\prime}\right)<W A M(U)\right)$

- For each criterion $i$, count the number $n_{\text {under_i }}^{\prime}\left(\right.$ resp. $n_{\text {over_i }}^{\prime}$ ) of times criterion $i$ is underestimated (resp. overestimated) in a vector of Higher $\mathrm{N}_{-}(U)$ (resp. Lower_ $\mathrm{N}(U)$ ), i.e., $U_{k(i)}^{\prime}$ takes the value just above $U_{k(i)}$ (resp. just below $U_{k(i)}$ ) in Higher_ $\mathrm{N}(U)$ (resp. Lower_ $\mathrm{N}(U)$ )

- Compute for each criterion i: $\operatorname{Diag}_{-}$under $(i)=\frac{n_{\text {under_i }^{\prime}}}{\mid \text { Higher_ } \mathrm{N}(U) \mid}$ (resp. $^{\text {Diag_over }(i)=\frac{n_{\text {over_i }}^{\prime}}{\mid \text { Lower_ }_{-} \mathrm{N}(U) \mid} \text { ) }}$

Diag_under $(i)$ gives the rate that an underestimation w.r.t. criterion $i$ be a potential cause of underestimation of the overall emergency degree of an operation (idem for overestimation).

Fig. 10 concerns underestimation diagnosis and Fig. 11 overestimation diagnosis for the base of operations in Fig. 4. A rate indicates the possibility a criterion is 
underestimated itself (resp. overestimated) when the overall emergency degree of the concerned operation is underestimated (resp. overestimated).

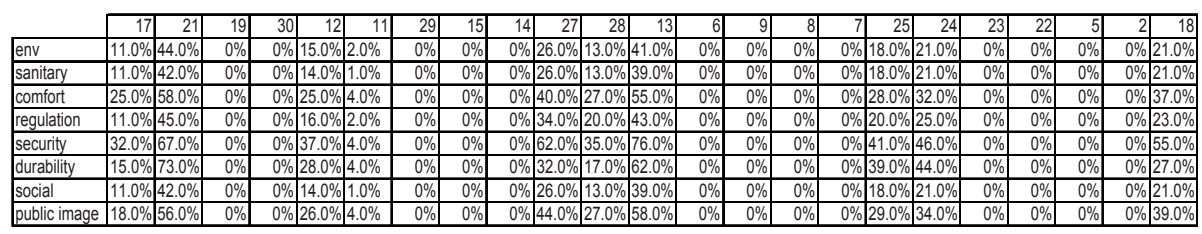

Fig. 8. Risk of overall underestimation of the operations induced by partial underestimations w.r.t. criteria.

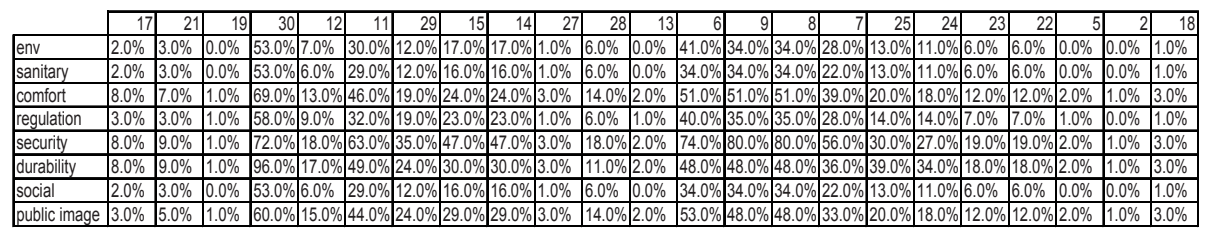

Fig. 9. Risk of overall overestimation of the operations induced by partial overestimations w.r.t. criteria.

\begin{tabular}{|c|c|c|c|c|c|c|c|c|c|c|c|c|c|c|c|c|c|c|c|c|c|c|c|}
\hline & 17 | & 211 & 19 & 30 & 12 & 111 & $29 \mid$ & 15 & \begin{tabular}{|l|l|}
14 & \\
\end{tabular} & & 28 & 13 & 6 & 9 & & 7 & 25 & 24 & \begin{tabular}{|l|l|}
23 \\
\end{tabular} & 22 & 5 & & 18 \\
\hline ability & & $57 \%$ & $0 \%$ & $0 \%$ & $67 \%$ & $100 \%$ & $0 \%$ & $0 \%$ & $80^{\circ}$ & $40 \%$ & $42 \%$ & $52 \%$ & $0 \%$ & $0 \%$ & $0 \%$ & $0 \%$ & $71 \%$ & $67 \%$ & $0 \%$ & $0 \%$ & $0 \%$ & $0 \%$ & $41 \%$ \\
\hline curity & $\%$ & $53 \%$ & $0 \%$ & $0 \%$ & $86 \%$ & $100 \%$ & $0 \%$ & $0 \%$ & - & $79 \%$ & $87 \%$ & $64 \%$ & $0 \%$ & $0 \%$ & $0 \%$ & $0 \%$ & $75 \%$ & $771 \%$ & $0 \%$ & $0 \%$ & $0 \%$ & $\%$ & \\
\hline Fort & $\%$ & $45 \%$ & $0 \%$ & $0 \%$ & $59 \%$ & $90 \%$ & $0 \%$ & $0 \%$ & $0 \%$ & $51 \%$ & $66 \%$ & $46 \%$ & $0 \%$ & $0 \%$ & $0 \%$ & $0 \%$ & $51 \%$ & $49 \%$ & $0 \%$ & $0 \%$ & $0 \%$ & $0 \%$ & $56 \%$ \\
\hline a & $55 \%$ & $44 \%$ & $0 \%$ & $0 \%$ & $62 \%$ & $90 \%$ & $80^{\circ}$ & $0 \%$ & $0 \%$ & $56 \%$ & $66 \%$ & $48 \%$ & $0 \%$ & $0 \%$ & $0 \%$ & $0 \%$ & $53 \%$ & $52 \%$ & $\%$ & $0 \%$ & $0 \%$ & $0 \%$ & $60 \%$ \\
\hline env & $35 \%$ & $35 \%$ & $0 \%$ & $0 \%$ & $36 \%$ & $45 \%$ & $0 \%$ & $0 \%$ & $0 \%$ & $33 \%$ & $33 \%$ & $35 \%$ & $0 \%$ & $0 \%$ & $0 \%$ & $0 \%$ & $33 \%$ & $33^{\circ}$ & $0 \%$ & $0 \%$ & $0 \%$ & \% & $33 \%$ \\
\hline regulati & $35 \%$ & $35 \%$ & $0 \%$ & $0 \%$ & $37 \%$ & $45 \%$ & $0 \%$ & $0 \%$ & $0 \%$ & $43 \%$ & $51 \%$ & $36 \%$ & $0 \%$ & $0 \%$ & $0 \%$ & $0 \%$ & $37 \%$ & $39 \%$ & $0 \%$ & $0 \%$ & $0 \%$ & $0 \%$ & $35 \%$ \\
\hline Ial & $33 \%$ & $33 \%$ & $0 \%$ & $0 \%$ & $33 \%$ & $33 \%$ & $0 \%$ & $0 \%$ & $0 \%$ & $33 \%$ & $33 \%$ & $33 \%$ & $0 \%$ & $0 \%$ & $0 \%$ & $0 \%$ & $33 \%$ & $33 \%$ & $0 \%$ & $0 \%$ & $0 \%$ & $\%$ & $33 \%$ \\
\hline sanitary & $33 \%$ & $33 \%$ & $0 \%$ & $0 \%$ & $33 \%$ & $33 \%$ & $\begin{array}{l}0 \% \\
\end{array}$ & $0 \%$ & $0 \%$ & $33 \%$ & $33 \%$ & $33 \%$ & $0 \%$ & $0 \%$ & $0 \%$ & $0 \%$ & $33 \%$ & \begin{tabular}{|l|l|}
$63 \%$ \\
\end{tabular} & \begin{tabular}{ll|}
$0 \%$ \\
\end{tabular} & $0 \%$ & $0 \%$ & 0\% & $33 \%$ \\
\hline
\end{tabular}

Fig. 10. Rates of causes of underestimation diagnoses.

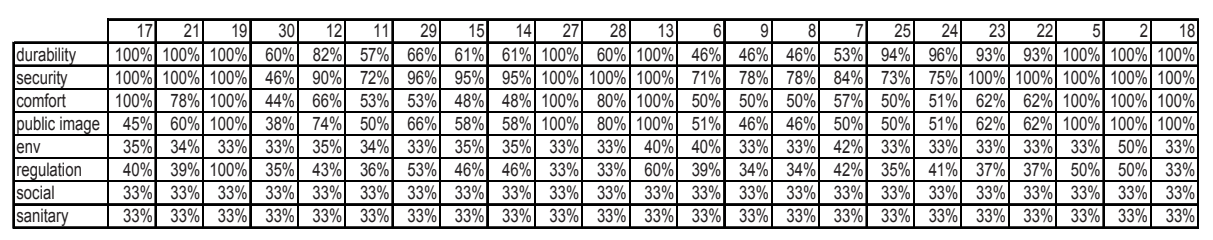

Fig. 11. Rates of causes of overestimation diagnoses.

\section{Conclusions}

In civil engineering, the culture of numbers is strongly developed. People commonly manipulate symbolic labels but attribute them numerical values when necessary without further care. A typical case is when aggregation procedures are required. We have proposed a methodology that enables 1) experts to express their judgement 
values in their own discrete semantic universe, 2) to convert the labels in adequate numerical values using the MACBETH method and clustering techniques, 3) to compute the WAM based aggregated value and convert it in return into the experts' semantic universe 4) to carry out a robustness analysis of the evaluation process to assess the risk of misclassification of the operations and to diagnose these misclassifications. This method is implemented in an IPS-SINERGIE-that supports decisions concerning maintenance operations planning by the motorway operator Escota.

\section{References}

1. Saaty, T.L.: The Analytic Hierarchy Process. McGraw-Hill, New York (1980)

2. Bana e Costa, C.A., Vansnick, J.C.: MACBETH - an interactive path towards the construction of cardinal value functions. International transactions in Operational Research, vol. 1, pp. 489-500 (1994)

3. Clivillé, V. : Approche Systémique et méthode multicritère pour la définition d'un système d'indicateurs de performance. Thèse de l'Université de Savoie, Annecy (2004)

4. Jullien, S., Mauris, G., Valet, L., Bolon, Ph.: Decision aiding tools for Animated film selection from a mean aggregation of criteria preferences over a finite scale. 11th Int. Conference on Information processing and Management of uncertainty in KnowledgeBased Systems, IPMU, Paris, France (2006)

5. Grabisch, M.: Representation of preferences over a finite scale by a mean operator. Mathematical Social Sciences, vol. 52, pp. 131-151 (2006)

6. Akharraz A., Montmain J., Mauris G.: A project decision support system based on an elucidative fusion system, Fusion 2002, 5th International Conference on Information Fusion, Annapolis, Maryland, USA (2002)

7. Akharraz A., Montmain J., Denguir A., Mauris G., Information System and Decisional Risk Control for a Cybernetic Modeling of Project Management. $5^{\text {th }}$ international conference on computer science (MCO 04), Metz, France, pp. 407-414 (2004). 